\title{
Politische Entscheidungsvorbereitung in der EU-27 plus am Beispiel der europäischen Innenpolitik
}

\author{
Matthias Oel und Juliane Rapp-Lücke*
}

In den vergangenen Jahren hat Europa die Nachkriegsphase endgültig überwunden. Nach dem Fall des ,Eisernen Vorhangs“ 1989 dauerte es 15 Jahre, bis am 1. Mai 2004 zehn Staaten, davon acht, die zuvor dem Warschauer Pakt angehörten, Mitglieder der Europäischen Union wurden. Nur kurze Zeit später folgten zum 1. Januar 2007 die Beitritte Rumäniens und Bulgariens, wodurch die Europäische Union vorerst auf 27 Mitgliedstaaten angewachsen ist. ${ }^{1}$ Seit dem 22. Dezember 2007 gehören die meisten neuen Mitgliedstaaten nunmehr auch dem Schengen-Raum an, sodass der freie Personen- und Warenverkehr ohne Grenzkontrollen über die vormalige Trennlinie zwischen Ost und West möglich ist.

Diese historisch herausragenden und politisch so bedeutsamen Entwicklungen haben auch das innere Gefüge der Europäischen Union nachhaltig verändert. Die politische Willensbildung und die legislativen Entscheidungsprozesse sind aufgrund der Anzahl der Mitgliedstaaten wesentlich komplexer geworden. Nicht wenige Politiker und Beamte klagen darüber, dass die Größe der Union zunehmend zu Lasten der Substanz der politischen Arbeit auf Ratsebene geht.

Die innerhalb von weniger als drei Jahren nahezu erfolgte Verdoppelung der Mitgliedstaaten der Europäischen Union von 15 auf 27 und mögliche weitere Erweiterungsschritte erfordern es, neue Wege der politischen Willensbildung im Verhältnis zwischen der Europäischen Kommission, den Mitgliedstaaten und dem Europäischen Parlament auszuprobieren. Dabei gilt es erstens, einer Entfremdung der politischen Ebene vom institutionellen Gefüge der Europäischen Union entgegenzuwirken. Es gilt zweitens, europäische Themen und die inhaltliche Auseinandersetzung mit ihnen stärker in das Bewusstsein der nationalen Parlamentarier und der europäischen Öffentlichkeit zu rücken. Und schließlich gilt es, bei alledem das vertraglich festgelegte institutionelle Gleichgewicht der Union, insbesondere das Initiativrecht der Europäischen Kommission und die Beteiligungsrechte des Europäischen Parlaments nicht zu unterminieren. Die Austarierung der divergierenden Interessen in diesem Dreieck ist nicht einfach, aber erforderlich.

Nachfolgend wird am Beispiel der europäischen Innenpolitik zunächst ein Überblick über die derzeitige Situation innerhalb und außerhalb des Rates gegeben. Anschließend wird als mögliches Modell für moderne Entscheidungsfindung in der EU-27 plus die unter deutscher Ratspräsidentschaft im Februar 2007 gegründete „Hochrangige Gruppe zur Zukunft der europäischen Innenpolitik“" und ihre Arbeit vorgestellt.

\footnotetext{
Ministerialrat Matthias Oel, EU-Direktor, Bundesministerium des Innern, Berlin.

Regierungsdirektorin Dr. Juliane Rapp-Lücke, LL.M. (NYU), EU-Direktorat, Bundesministerium des Innern, Berlin.

Dieser Beitrag gibt ausschließlich die persönliche Meinung der beiden Verfasser wieder.

1 Im Oktober 2005 wurden Beitrittsverhandlungen mit Kroatien und der Türkei eröffnet. Der ehemaligen jugoslawischen Republik Mazedonien wurde im Dezember 2005 der Kandidatenstatus verliehen. Als potenzielle Beitrittskandidaten hat die Europäische Union Albanien, Bosnien und Herzegowina, Montenegro und Serbien benannt.
} 


\section{Politische Willensbildung auf Ratsebene am Beispiel des Rates für Justiz und Inneres: Anspruch und Wirklichkeit}

$\mathrm{Ob}$ auf regionaler, nationaler oder internationaler Ebene: wenn es um politische Willensbildung und Entscheidungsprozesse geht, sind sich alle Beteiligten und Beobachter schnell einig. Abläufe und Verfahren sollten möglichst transparent und effizient, die eine Entscheidung vorbereitenden Diskussionen gut vorbereitet, substanziiert und zielorientiert sein.

Wenngleich diese Ziele unumstritten sind, bleiben der Weg dorthin und mögliche Lösungsansätze im Einzelfall schwierig. Dies gilt vor allem dann, wenn die gesetzten Rahmenbedingungen, wie in der EU-27 plus, komplex sind. Hier klaffen Anspruch und Wirklichkeit zunehmend auseinander.

Ein kurzer Überblick über eine Sitzung des Rates der Justiz- und Innenminister verdeutlicht dies: Der große, fensterlose Ratssaal im Ratsgebäude in Brüssel bietet Platz für circa 150 Vertreter von Ratspräsidentschaft, Europäischer Kommission, Ratssekretariat sowie nationalen Delegationen (Minister beziehungsweise Staatssekretär, Botschafter und drei Berater). Hinzu kommen um den achteckig aufgestellten Ratstisch über 50 Dolmetscher für die insgesamt 23 Amtssprachen. Vor sich haben die Minister Monitore, da sie die Mimik des jeweils sprechenden Kollegen aufgrund der Saalgröße sonst nicht erkennen könnten. Zu relativ unproblematischen Tagesordnungspunkten führen im Regelfall Vorsitz und Kommission kurz ein, einige Minister geben Statements ab und der Vorsitz fasst abschließend zusammen. Komplexere Tagesordnungspunkte erfordern eine Tischumfrage, die bei einer durchschnittlichen Redezeit von nur drei Minuten insgesamt etwa eineinhalb Stunden dauert.

Diese Skizze zeigt, dass Ratssitzungen heute kaum mehr dazu dienen (können), auf Ministerebene substanziell und kontrovers zu verhandeln. Die Abläufe der Sitzungen sind zwangsläufig schematisch und erfordern eine nahezu abschließende Vorbereitung durch die nachgeordneten Ratsgremien.

Beim informellen Ministertreffen unter finnischem Vorsitz in Tampere im September 2006 diskutierten die Innenminister über die Evaluierung des sogenannten „Haager Programms zur Stärkung von Freiheit, Sicherheit und Recht in der Europäischen Union“, dem geltenden Mehrjahresprogramm, das Ziele der europäischen Innenpolitik festlegt. Im Rahmen dieser Debatte wurde überraschend deutlich, wie unzufrieden die meisten Minister mit der Länge der Entscheidungsprozesse im Rat und dem unflexiblen, langatmigen Ablauf der Ratstagungen sind. ${ }^{2}$ Die Frustration unter den Politikern hat in den vergangenen Jahren auch dazu geführt, dass manche Minister nicht mehr selbst an den Sitzungen teilnehmen, sondern sich teilweise regelmäßig von Staatssekretären oder Botschaftern vertreten lassen. Diese Entwicklung führt zwangsläufig dazu, dass eine wirklich politische Erörterung von Vorhaben auf europäischer Ebene nicht mehr hinreichend gewährleistet ist und die Legitimität von Ratsentscheidungen abnimmt.

Die Frage stellt sich, welche Alternativen es gibt, um die Entscheidungsvorbereitung und -findung in der Europäischen Union zu straffen und wieder politischer zu gestalten.

\section{Politische Entscheidungsvorbereitung außerhalb des Rates am Beispiel der europä- ischen Innenpolitik}

Die dargestellte Tendenz zur ,Entpolitisierung، der Ratsarbeit hat schon zu Zeiten der EU der 15 dazu geführt, dass sich parallel zu den Ratsstrukturen Sondergruppen gebildet haben,

2 Siehe Finnische Ratspräsidentschaft: More effective decision-making in Justice and Home Affairs important to deepen practical cooperation in security issues, Presseerklärung vom 22.09.2006, abrufbar unter: http:// www.eu2006.fi/news_and_documents/press_releases/vko38/en_GB/168911/ (letzter Zugriff: 11.06.2008). 
die politische Substanzdebatten im kleineren Kreis erlauben. Die Arbeit solcher Gruppen soll im Folgenden an den Beispielen des sogenannten Salzburg-Forums (auch SalzburgGruppe) und der G6-Gruppe verdeutlicht werden. ${ }^{3}$

\section{Das Salzburg-Forum}

Das Salzburg-Forum wurde im Jahr 2000 auf Initiative Österreichs gegründet. Bis 2005 gehörten dieser regionalen Kooperation im Bereich der inneren Sicherheit mit Österreich die Staaten Polen, Slowenien, Ungarn, Slowakische Republik und Tschechische Republik an. 2006 wurden Rumänien und Bulgarien aufgenommen. Ferner nimmt seit 2006 auch Kroatien als Beobachter teil. Auf Ministerebene tagt die Gruppe zweimal jährlich. 2006 einigten sich die Minister entsprechend der Gepflogenheiten im Rat auf einen halbjährlichen Vorsitzwechsel. Zurzeit übt Ungarn den Vorsitz aus.

Das Salzburg-Forum hat zur operativen Zusammenarbeit Unterstrukturen gebildet. So gibt es derzeit Arbeitsgruppen zu den Themen Zeugenschutz, Herkunftslandinformation, Verkehrspolizei, Schengen-Evaluierung, DNA-Datenaustausch und Großereignisse. ${ }^{4}$

\section{Die G6-Gruppe}

Im Jahr 2003 gründeten Frankreich, das Vereinigte Königreich, Italien, Spanien und Deutschland die sogenannte G6-Gruppe zunächst als G5, um in einem informellen Kreis Fragen der europäischen Innenpolitik, insbesondere der Migration, Terrorismusbekämpfung und polizeilichen Zusammenarbeit zu diskutieren. 2006 wurde Polen nach dessen Beitritt zur Europäischen Union als sechstes Mitglied aufgenommen, das damit sowohl am Salzburg-Forum als auch an der G6-Gruppe teilnimmt.

Auch die G6-Gruppe tagt zweimal jährlich auf Ministerebene mit halbjährlich wechselnden Präsidentschaften. Seit 1. Juli 2008 hat Frankreich den Vorsitz inne. 2009 folgen im ersten Halbjahr Deutschland und im zweiten Halbjahr das Vereinigte Königreich.

Zur Vorbereitung der Ministersitzungen veranstaltet der jeweilige Vorsitz Treffen auf Generaldirektorenebene, im Verlaufe derer auch der Stand der Arbeiten der laufenden G6Projekte diskutiert wird. Die zurzeit 18 Projekte in den Bereichen Terrorismusbekämpfung, Bekämpfung der organisierten Kriminalität und Migration resultieren aus den Schlussfolgerungen der Ministertreffen im Jahr 2006 in Heiligendamm ${ }^{5}$ und Stratford-upon-Avon. ${ }^{6}$ Diskussionen darüber finden in Ad-hoc-Arbeitsgruppen auf Expertenebene statt.

3 Darüber hinaus bestehen weitere (regionale) Gruppierungen. Zu nennen ist insbesondere die „Task Force on Organised Crime in the Baltic Sea Region“ auch „Visby-Gruppe“ genannt. Diese unterscheidet sich allerdings insofern von den übrigen dargestellten Gruppen, als es sich um eine Arbeitsgruppe des Ostseerates handelt. Ihr gehören neben Mitgliedstaaten der Europäischen Union auch Norwegen, Island und Russland an. Die Europäische Kommission hat Beobachterstatus. Die Visby-Gruppe hat sich zu einem wichtigen Forum für die operative, praktische Zusammenarbeit bei der Bekämpfung organisierter Kriminalität im Ostseeraum entwickelt. Mitglieder sind persönliche Vertreter der teilnehmenden Regierungen. Unterhalb dieser Ebene sind ein ,Operationeller Ausschuss' sowie einzelne Expertengruppen eingerichtet worden. Zur Visby-Gruppe siehe die Internetseite der Task Force unter: http://www.balticseataskforce.ee/home.nsf/pages/indexeng.html (letzter Zugriff: 11.06.2008).

4 Vgl. österreichisches Bundesministerium für Inneres: Salzburg-Gruppe. Europäische Erfolgsgeschichte, in: Öffentliche Sicherheit 3-4/2007, S. 42-44.

5 Bundesministerium des Innern: Schlussfolgerungen des Treffens der Innenminister von Frankreich, Italien, Polen, Spanien, Deutschland und dem Vereinigten Königreich in Heiligendamm, 22./23. März 2006, Pressemitteilung, abrufbar unter: http://www.bmi.bund.de/nn_334158/Internet/Content/Nachrichten/Pressemitteilungen/2006/03/Innenministertreffen__Heiligendamm.html (letzter Zugriff: 11.06.2008).

6 Home Office: Conclusions of the October 2006 Meeting of the interior Ministers of the UK, France, Germany, Italy, Poland and Spain, 25./26. Oktober 2006, Pressemitteilung, abrufbar unter: http://press.homeoffice. gov.uk/press-releases/g6-meeting-conclusions (letzter Zugriff: 11.06.2008). 


\section{,Salzburg-Gruppe meets G6'?}

Für das erste Halbjahr 2007 wurde ein gemeinsames Treffen der Salzburg- und G6Gruppe unter österreichischem Vorsitz angeregt. Da ein solches Treffen nahezu die Hälfte, genauer zwölf der EU-Mitgliedstaaten vereint hätte, kamen die G6-Innenminister jedoch überein, dass der polnische Innenminister als Mitglied beider Gruppen die Interessen der G6 allein mit vertreten solle.

\section{Bewertung}

„Was in einer EU der 27 immer schwieriger wird, soll hier im Kleinen vorexerziert werden, um dann auf europäischer Ebene gemeinsame Positionen einbringen zu können. Diesen Zielen hat sich das Forum Salzburg verschrieben [...]."7

Dieses Fazit aus einem Artikel über die Salzburg-Gruppe belegt, dass dieses Forum nicht nur zur verbesserten regionalen Abstimmung und zur Stärkung der Rolle dieser Region in der Europäischen Union gegründet wurde. Ziel ist es, auch außerhalb des Rates auf Experten- und Ministerebene im kleineren Kreis zu diskutieren und politische Entscheidungen vorzubereiten.

Für die G6-Gruppe ist letzteres sicherlich ebenfalls einschlägig. An die Stelle des regionalen Ansatzes tritt hier jedoch das Kriterium ,größte Mitgliedstaaten`. Anders als bei dem nachbarschaftlich kooperierenden Salzburg-Forum führt die Zusammensetzung der G6Gruppe bei den übrigen Mitgliedstaaten der Europäischen Union allerdings oftmals, meist aber hinter vorgehaltener Hand, zu Kritik und gar zu Unmut. Gefürchtet wird, dass die ,sechs Großen“ versuchen könnten, ein ,EU-Direktorium‘ zu bilden, das versuchen könnte, die Entscheidungsprozesse innerhalb des Rates zu dominieren und gleichzeitig das bestehende institutionelle Gefüge zu verändern.

Die Praxis seit Gründung der G5(6) im Jahr 2003 hat jedoch gezeigt, dass diese Befürchtungen wohl unbegründet sind. Zu unterschiedlich sind die Interessen der regional nicht zusammenhängenden Mitglieder, zu selten die Ministertreffen einmal pro Semester, um tatsächlich ein abgestimmtes Handeln im Rat über den Einzelfall hinaus zu ermöglichen. Gelungen ist allerdings zum Beispiel Ende 2006 die Einbringung einer Initiative durch den damaligen britischen G6-Vorsitz in den Rat auf der Grundlage eines vom deutschen Bundesminister des Innern Dr. Wolfgang Schäuble und dem damaligen französischen Innenminister Nicolas Sarkozy beim G6-Treffen im Oktober 2006 vorgestellten deutsch-französischen Strategiepapiers für eine neue europäische Migrationspolitik. ${ }^{8}$ In der Folge hat der Europäische Rat Schlussfolgerungen verabschiedet, in denen die Staats- und Regierungschefs unter anderem dazu auffordern, nach Mitteln und Wegen zu suchen, wie die zirkuläre und temporäre Migration erleichtert werden kann. ${ }^{9}$ Insgesamt bleiben aber die Befürchtungen hinsichtlich einer Dominanz der G6-Gruppe auf die Ratsarbeit größer als ihr tatsächlicher Einfluss. ${ }^{10}$

Obwohl G6-Gruppe und Salzburg-Forum zu für den Zusammenhalt der Europäischen Union positiven regelmäßigen Treffen der beteiligten Innenminister in informeller Atmosphäre und damit zu tiefergehenden und substanzielleren Diskussionen führen, so haben sie doch einen Nachteil: Beide Gruppierungen wollen unter anderem die politische Entschei-

7 Bundesministerium für Inneres: Salzburg-Gruppe, 2007, S. 44.

8 Vgl. Home Office: Conclusions of the October 2006 Meeting, 2006.

9 Rat der Europäischen Union: Europäischer Rat (Brüssel), 14./15. Dezember 2006, Schlussfolgerungen des Vorsitzes, Ratsdokument 16879/06 (CONCL3), S. 9.

10 Dies ist gerade angesichts der im G6-Rahmen gebildeten umfangreichen Unterstrukturen zur Verfolgung von Projekten festzuhalten. Vergleichbar dazu hat auch das Salzburg-Forum mit seinen zahlreichen Arbeitsgruppen ein institutionelles Gefüge entwickelt. 
dungsfindung im Rat beeinflussen und vorbereiten, laufen dabei aber Gefahr, Ratsdiskussionen zu duplizieren. Zugleich agieren sie weitgehend unabhängig von den Ratsstrukturen; jedenfalls im Rahmen von G6 wird dabei die Kommission zumindest nur unregelmäßig beteiligt. Wenn dies zusätzlich dazu führt, dass einzelne Innenminister zwar an Treffen dieser Gruppen, aber nicht mehr an Ratssitzungen teilnehmen, so wird die zentrale Stellung des Rates als Legislativ- und politisches Entscheidungsorgan der Europäischen Union geschwächt.

Beide Gruppen verfolgen ferner weder nach ihren Zielsetzungen noch ihrer bisherigen Praxis den umgekehrten Ansatz, im kleinen Kreis oder auf regionaler Ebene darüber zu beraten, wie europäische Entscheidungen bestmöglich umgesetzt und flankiert werden können. Die Arbeit der Europäischen Union wird aber gerade dann bürgernäher und transparenter, wenn nicht nur versucht wird, die Ratsarbeit im Vorfeld zu beeinflussen, sondern auch darüber diskutiert wird, wie europäische Vorgaben unter Berücksichtigung der Besonderheiten einzelner Mitgliedstaaten oder der Region konkret umgesetzt werden sollen.

Zusammenfassend kann festgestellt werden, dass sich Gruppierungen wie die Salzburgoder die G6-Gruppe positiv auf die Kontaktpflege, auf die Abstimmung zwischen den beteiligten Ministern und auf die regionale Zusammenarbeit auswirken. Dabei fungieren sie als Relais zwischen der europäischen und der mitgliedstaatlichen Ebene. Bei Verfolgung eines zu ambitiösen Ansatzes bergen sie jedoch durch den Ausschluss anderer Mitgliedstaaten und die punktuelle oder fehlende Einbindung der Kommission auch die Gefahr, die politische Meinungsbildung im Rahmen der Europäischen Union zu ,zerfasern ‘ und damit im Ergebnis zu einer Schwächung der Ratsstrukturen und des institutionellen Gefüges der Europäischen Union insgesamt beizutragen.

\section{Pilotprojekt ,Zukunftsgruppe' für die politische Entscheidungsvorbereitung in der EU-27 plus}

\section{Herausforderung}

Die politische Entscheidungsvorbereitung und -findung in der Europäischen Union steht also zurzeit vor einem Dilemma: Einerseits ist es schwierig, die Arbeiten des aus 27 Mitgliedstaaten und der Kommission bestehenden Rates entscheidend zielorientierter und effizienter zu gestalten. Andererseits sollten mögliche Lösungswege aus europapolitischer Sicht weder zu einer willkürlichen Auswahl von Teilnehmern führen noch für die Entscheidungsvorbereitung zentrale Akteure gleichsam , ausklammern'. Eine mögliche Lösung des Dilemmas setzt also voraus, dass ein kleinerer Kreis von Mitgliedstaaten und die Kommission Vorüberlegungen anstellen, dass die nicht-teilnehmenden Mitgliedstaaten dieses Vorgehen mittragen und dass es die ,Brüsseler Architektur der Entscheidungsfindung ' nicht in Frage stellt.

\section{Initiative unter deutscher Ratspräsidentschaft}

Der deutsche Bundesminister des Innern Dr. Wolfgang Schäuble und der damalige für Innen- und Justizpolitik zuständige Vizepräsident der Kommission Franco Frattini unterbreiteten beim informellen Ministertreffen unter deutscher Ratspräsidentschaft im Januar 2007 in Dresden den Vorschlag, einen neuen Weg der politischen Entscheidungsvorbereitung einzuschlagen: Eine kleine, aber repräsentative Gruppe sollte langfristige Vorüberlegungen anstellen, welche politischen Prioritäten in der europäischen Innenpolitik bis 2014 gesetzt werden sollten. Diese grundsätzliche Ausrichtung hatte der zukünftige deutsche Vorsitz zuvor in Einzelgesprächen auf politischer Ebene mit allen übrigen Mitgliedstaaten und der Kommission vorbereitet. 
Hintergrund dieses Vorschlags war, dass bis Ende 2009 die Ziele der gemeinsamen europäischen Innenpolitik noch durch das geltende europäische Mehrjahresprogramm, das „Наager Programm zur Stärkung von Freiheit, Sicherheit und Recht", definiert werden. Die Innenminister waren sich darüber einig, dass nach dessen Ablauf ein neues Mehrjahresprogramm für den Zeitraum 2010 bis 2014 verabschiedet werden sollte. Aufgabe der Gruppe sollte es sein, möglichst frühzeitig Empfehlungen zu Inhalt und Prioritäten des künftigen ,Post-Haager-Programms` zu erarbeiten.

Um eine willkürliche Auswahl von Mitgliedern zu vermeiden, schlugen Bundesminister Dr. Schäuble und Vizepräsident Frattini vor, dass das objektive Kriterium für die Gruppenmitgliedschaft grundsätzlich die Abfolge der Ratspräsidentschaften sein sollte. Den Ko-Vorsitz der Gruppe sollten die jeweilige amtierende Ratspräsidentschaft und die Kommission führen.

\section{Struktur und Besonderheiten der sogenannten ,Zukunftsgruppe'}

In Dresden und bei einem gemeinsamen Abendessen im Februar 2007 einigten sich die Innenminister und die Kommission über die Zusammensetzung der Gruppe. Mitglieder der sogenannten ,Zukunftsgruppe“ waren neben Kommissions-Vizepräsident Frattini beziehungsweise seit Frühjahr 2008 Vizepräsident Jacques Barrot die Innenminister der damals amtierenden und der nachfolgenden Trio-Präsidentschaft - also von Deutschland, Portugal und Slowenien sowie von Frankreich, der Tschechischen Republik und Schweden. Ferner nahm ein jeweils wechselnder Vertreter der darauffolgenden Trio-Präsidentschaft aus Spanien, Belgien und Ungarn teil. Zusätzlich entsandte die Gruppe der Mitgliedstaaten mit einem ,Common Law'-System einen Beobachter aus dem Vereinigten Königreich. Für das Europäische Parlament nahm der jeweilige Vorsitzende des Ausschusses für bürgerliche Freiheiten, Sicherheit und Recht als Beobachter teil. Auch das Generalsekretariat des Rates war mit Beobachterstatus vertreten.

Diese somit nur elf Mitglieder umfassende Gruppe spiegelt das ,Gravitationszentrum ‘ der Europäischen Union, bestehend aus der Kommission, den amtierenden beziehungsweise zukünftigen Präsidentschaften als zentralen Verhandlungsführern im Rat sowie dem Europäischen Parlament, wieder. Die Trio-Präsidentschaften bilden gleichzeitig eine repräsentative und ausgewogene Mischung aus alten und neuen, nördlichen und südlichen Mitgliedstaaten.

Die jeweiligen Amtsinhaber nahmen ad personam, also nicht in ihrer amtlichen Eigenschaft teil. Es wurde ausdrücklich festgelegt, dass die Gruppe nicht in die formalen Entscheidungs- und Gremienstrukturen der Union eingegliedert sei.

Trotz dieses informellen Charakters der Gruppe hatten die nicht-teilnehmenden Mitgliedstaaten selbstverständlich einen Anspruch darauf, regelmäßig und transparent über deren Arbeiten informiert zu werden. Dies ermöglichte es ihnen zugleich, zu den laufenden Arbeiten beizutragen. Insoweit übte das Generalsekretariat des Rates als Beobachter eine Brückenfunktion aus. Regelmäßig lud der Vorsitz teils aus dem Kreis der nicht-teilnehmenden Mitgliedstaaten, teils auch darüber hinausgehend Gäste als Experten ein. So haben die Minister Luc Frieden (Luxemburg), Giuliano Amato (Italien) und Günther Platter (Österreich) sowie der ehemalige Kommissar António Vitorino oder der Terrorismuskoordinator des Rates Gilles de Kerchove an einzelnen Sitzungen teilgenommen. Transparenz wurde ferner durch folgende Maßnahmen hergestellt: Die Diskussionspapiere und Protokolle der Sitzungen wurden allen Ratsmitgliedern zugänglich gemacht. Zudem berichteten Präsidentschaft und Kommission im Rahmen von informellen Essen mündlich über den Fortgang der Beratungen. Zur Halbzeit der Gruppensitzungen legte die slowenische Präsidentschaft Anfang 2008 einen Zwischenbericht vor, der die wesentlichen Themen und Diskussionsbeiträge zusammenfasste. 
Innerhalb der Bundesrepublik wurden in gleicher Weise fachlich betroffene Bundesressorts sowie die Innenministerien der Länder laufend informiert. Innen- und Europaausschuss sowie Arbeitsgruppen von Fraktionen des Deutschen Bundestages wurden mündlich unterrichtet. Um auch der Öffentlichkeit eine Verfolgung der Arbeiten der Gruppe zu ermöglichen, sind alle relevanten Dokumente auf der Internetseite des Bundesministeriums des Innern veröffentlicht. ${ }^{11}$

\section{Arbeit der ,Zukunftsgruppe‘}

Während ihres ersten Treffens im Mai 2007 in Eltville verabschiedete die Gruppe ihre ,Terms of Reference', in denen sie die genannten Grundsätze festschrieb und eine ,SherpaGruppe " zur Vorbereitung der Sitzungen auf Ministerebene und zur Erarbeitung des Abschlussberichts einsetzte. ${ }^{12}$

Die Gruppe hat sich unter deutscher, portugiesischer und slowenischer Präsidentschaft insgesamt sechs Mal getroffen. Inzwischen liegt der Abschlussbericht vor, der die Empfehlungen der Gruppenmitglieder für das kommende Mehrjahresprogramm enthält. ${ }^{13}$ Er wurde am 7. Juli 2008 beim informellen Ministertreffen in Cannes den Ratsmitgliedern vorgestellt und mit ihnen diskutiert. Unmittelbar im Anschluss wurde der Text der Öffentlichkeit zugänglich gemacht. Es wäre wünschenswert und entspräche dem Ziel der Gruppe, wenn im Herbst sowohl der Ausschuss des Europäischen Parlaments für bürgerliche Freiheiten, Justiz und Inneres sowie in Deutschland Europa- und Innenausschuss des Bundestages, die zuständigen Gremien der Länder, den Bericht behandeln würden. Die Debatte im politischen Raum würde nicht zuletzt von einer wissenschaftlich fundierten Bewertung und Diskussion des Berichts erheblich profitieren.

Vizepräsident Jacques Barrot hat angekündigt, dass die Kommission ihren formellen Vorschlag für das ,Post-Haager-Programm‘ frühestens im März 2009 vorlegen wird. Angesichts dieses Zeitplans ist davon auszugehen, dass das neue Mehrjahresprogramm für Justiz und Inneres unter schwedischem Ratsvorsitz im Herbst 2009 vom Europäischen Rat verabschiedet wird.

\section{Schlussfolgerung und Ausblick}

Anders als die oben dargestellten Gruppen G6 und Salzburg-Forum führte die Zukunftsgruppe das ,Gravitationszentrum ' der Europäischen Union bestehend aus Präsidentschaften, Kommission und Parlament zusammen. Die Gruppe war damit auch die konsequente Fortentwicklung des Trio-Präsidentschafts-Gedankens, durch den mehr Kontinuität und Kohärenz in die Europapolitik gebracht werden soll. Hervorzuheben ist auch, dass der Grundgedanke der Zukunftsgruppe eine rollierende Mitgliederstruktur war, die zudem eine geographische und interessenorientierte Ausgewogenheit gewährleistete. Weiterhin arbeitete die Gruppe im Gegensatz zu G6 und dem Salzburg-Forum projektorientiert mit einem zeitlich begrenzten Mandat und mit Billigung aller Mitgliedstaaten. Schließlich verfügt das mit der Zukunftsgruppe verfolgte Modell auch über einen größeren Wirkungsradius, da von der Gruppe erwartet wird, ihre Empfehlungen auf europäischer Ebene unmittelbar dem Rat, der Kommission und dem Europäischen Parlament zur Verfügung zu stellen.

11 Siehe Informationen des Bundesministeriums des Innern zur hochrangigen Gruppe zur Zukunft der europäischen Innenpolitik unter: http://www.bmi.bund.de/nn_163598/Internet/Navigation/DE/Themen/Europa/ZukunftsGruppe/ZukunftsGruppe_node.html (letzter Zugriff: 08.07.2008).

12 Ebenda.

13 Ebenda. 
Im Unterschied zur normalen Ratsarbeit zeichnete sich der Auftrag der Gruppe dadurch aus, dass über Tagespolitik und konkrete Vorhaben hinaus auch fachübergreifend auf politischer Ebene über zukünftige Herausforderungen und ihre Bewältigung diskutiert wurde.

Im Verhältnis zwischen Kommission und Mitgliedstaaten brachte die Zukunftsgruppe für beide Seiten Vorteile: Für den zuständigen Vizepräsidenten der Kommission bot der KoVorsitz angesichts der wachsenden Rolle informeller Foren der Mitgliedstaaten die Möglichkeit, die Präsenz der Kommission zu verstärken. Vor der Vorlage des Vorschlags für ein ,Post-Haager-Programm“ konnte die Kommission in der Gruppe einen Einblick in die politischen Prioritäten einer repräsentativen Gruppe von Ministern erhalten, die zudem während der Verhandlungen über den Kommissionsvorschlag den Vorsitz im Rat führen. Die Mitgliedstaaten wiederum, die gerade im Bereich der die staatliche Souveränität besonders betreffenden Innenpolitik ihre starke Stellung behaupten wollen, konnten die Gelegenheit nutzen, frühzeitig vor Erarbeitung des Vorschlags der Kommission gemeinsam mit dem verantwortlichen Kommissar Meinungen auszuloten.

Die persönliche Mitgliedschaft ohne formale Bindung an die jeweiligen Regierungen beziehungsweise das Kollegium und die geringe Größe der Gruppe, die einen lockereren und vertraulicheren Rahmen für die Diskussionen bot, haben sich insgesamt positiv im Vergleich zur üblichen Ratsarbeit ausgewirkt. Dies galt übrigens nicht nur für die Ministerebene, sondern auch für die fachübergreifend zusammengesetzte Sherpa-Gruppe.

Wie jedes neue Projekt hat die Zukunftsgruppe jedoch auch vielfach Skepsis und Bedenken ausgelöst.

In der Kommission wurde vor allem auf der Fachebene Sorge um eine Aushöhlung des Initiativrechts geäußert. Doch ihr rein informeller Charakter und die Mitgliedschaft ad personam verdeutlichen, dass die Zukunftsgruppe das Initiativrecht der Kommission weder antasten sollte noch antasten konnte. Im Übrigen erscheint eine solche Befürchtung jedenfalls vor dem Hintergrund der Alternative, wonach der informelle Gedankenaustausch über die zukünftige Gestaltung der Politik sonst jedenfalls zum Teil ohne die Kommission in regionalen Gruppierungen geführt würde, nachrangig. Dies gilt umso mehr, als die Kommission im Zusammenhang mit ihren Bemühungen um bessere Rechtsetzung bereits dazu übergegangen ist, in informellen Reflexionsgruppen vor der Erarbeitung ihres formellen Vorschlags mit einem Teil der Mitgliedstaaten und interessierten Kreisen zu diskutieren. ${ }^{14}$

Bei den nicht-teilnehmenden Mitgliedstaaten wurde vor allem zu Beginn der Arbeiten Sorge um die Transparenz und die Möglichkeit der Mitarbeit durch externe Beiträge laut. Diese Bedenken konnten durch die oben dargestellte ständige Unterrichtung und insbeson-

14 Die im Januar 2005 vom für Unternehmen und Industrie zuständigen Vizepräsidenten der Europäischen Kommission Günter Verheugen einberufene hochrangige Gruppe „CARS 21“ („Competitive Automotive Regulatory System for the 21st Century“) setzte sich aus weiteren Vertretern der Europäischen Kommission (Vizepräsident Jacques Barrot, Verkehr; Kommissar Stavros Dimas, Umwelt), der Mitgliedstaaten (Wirtschafts-, Umwelt- bzw. Verkehrsminister aus Deutschland, dem Vereinigten Königreich, Frankreich, Italien und der Tschechischen Republik), des Europäischen Parlaments sowie von Industrie, Gewerkschaften, Nichtregierungsorganisationen und Verbrauchern zusammen. Die Gruppe hatte den Auftrag, Empfehlungen für die zukünftige Politik und den Rechtsrahmen im Automobilbereich zu entwickeln. Weitere Informationen zu dieser Gruppe und der am 12. Dezember 2005 veröffentlichte Bericht sind abrufbar unter: http://ec.europa.eu/ enterprise/automotive/pagesbackground/competitiveness/cars21.htm (letzter Zugriff: 11.06.2008).

Auch das im September 2006 einberufene „Pharmaceutical Forum“ setzt sich aus Vertretern der Europäischen Kommission (Vizepräsident Günter Verheugen und Gesundheitskommissar Markos Kyprianou, nunmehr Kommissarin Androulla Vassiliou), Ministern aller teilnahmewilligen Mitgliedstaaten, drei Vertretern des Europäischen Parlaments, Vertretern von Interessenverbänden sowie hohen Repräsentanten der Pharmaindustrie zusammen. Ziel ist es, die Wettbewerbsfähigkeit der pharmazeutischen Industrie und ihren Beitrag zu Zielen im Bereich des Sozialen und der öffentlichen Gesundheit zu verbessern. Weitere Informationen unter: http:// ec.europa.eu/enterprise/phabiocom/comp_pf_en.htm (letzter Zugriff: 11.06.2008). 
dere durch den von der slowenischen Präsidentschaft vorgelegten Zwischenbericht wohl ausgeräumt werden. Die Beseitigung solcher Bedenken ist umso wichtiger, als davon die Akzeptanz eines solchen Forums auf europäischer und nationaler Ebene abhängt. Letztlich ist eine hinreichende Transparenz damit Voraussetzung für eine etwaige zukünftige Nutzung des Modells.

Zusammenfassend lässt sich feststellen, dass die Zukunftsgruppe mit der Vorlage ihres Berichts ihre wesentlichen Zwecke (Erörterung eines für mehrere Jahre wegweisenden Projekts und Ermöglichung eines öffentlichen Diskurses im Vorfeld der Erarbeitung eines formellen Vorschlags durch die Kommission und damit Herstellung von mehr Öffentlichkeit der Ratsarbeit) erreicht hat. Ob dieser neue Weg der Vorbereitung politischer Entscheidungen innerhalb der Brüsseler Architektur ein Einzelfall bleibt oder aber in Zukunft bei herausragenden politischen Projekten wiederholt wird, bleibt abzuwarten. Die Tatsache, dass die Justizminister unter portugiesischer Präsidentschaft im zweiten Halbjahr 2007 ebenfalls eine Hochrangige Gruppe zur Zukunft der europäischen Justizpolitik gründeten, zeigt, dass dies nicht ausgeschlossen ist.

Auch unabhängig vom Modell der Zukunftsgruppe ist ein partnerschaftliches Zusammenwirken zwischen Mitgliedstaaten und Kommission bei der politischen Entscheidungsvorbereitung in der EU-27 plus wünschenswert. Dies gilt gerade in dem hinsichtlich der staatlichen Souveränität besonders sensiblen Bereich der europäischen Innenpolitik.

Der Kommission kommt in der sogenannten ersten Säule für Rechtsakte das alleinige Initiativrecht zu. Dabei ist sie aber nicht in gleichem Maße wie eine nationale Regierung der Mehrheit eines sie tragenden und von einem Staatsvolk gewählten Parlaments politisch verantwortlich. Diese Besonderheit des institutionellen Gefüges der Europäischen Union erfordert umso mehr, dass sich Kommission, Rat und Parlament gegenseitig vertrauen. Sie müssen im Verhältnis zueinander eine politische Willensbildung ermöglichen, die den naturgemäß widerstreitenden Interessen ausreichend Raum verleiht. Damit würde gleichzeitig kompensiert, dass der Rat mit der gestiegenen Anzahl der Mitgliedstaaten heterogener geworden ist und die Mitgliedstaaten dadurch gegenüber der Kommission an politischen Einflussmöglichkeiten eingebüßt haben. Diese faktische Kräfteverschiebung würde sich mit Inkrafttreten des Vertrags von Lissabon, der das Initiativrecht der Kommission stärken und nahezu flächendeckend Mehrheitsentscheidungen einführen würde, noch ausweiten.

Der durch die Erweiterung und das hoffentlich baldige Inkrafttreten des Vertrags von Lissabon fortschreitende europäische Integrationsprozess erfordert also auf allen Seiten den Mut, neue Wege der politischen Entscheidungsvorbereitung zu erproben, die das Initiativrecht der Kommission nicht beeinträchtigen, aber die Interessen im institutionellen Dreieck zwischen Kommission, Mitgliedstaaten und Parlament ausbalancieren. 